\title{
Distributed Aperiodic Model Predictive Control for Multi-Agent systems
}

\author{
Kazumune Hashimoto, Shuichi Adachi and Dimos V. Dimarogonas * *ł‡
}

\begin{abstract}
In this paper, we propose an aperiodic formulation of Model Predictive Control for distributed agents with additive bounded disturbances. In the proposed method, each agent solves an Optimal Control Problem only when certain control performances cannot be guaranteed according to certain triggering rules. This could lead to the reduction of energy consumption and the alleviation of over-usage of communication resources. The triggering rules are derived for both event-triggered and self-triggered formulation. Our proposed method is also verified through a simulation example.
\end{abstract}

\section{Introduction}

The analysis of formation control of cooperating autonomous vehicles is an important area of research for the last 3 decades. This has been motivated for many years since it increases the efficiency and lowers the overall loads and costs by completing tasks together working as a team of agents. The most common approach to the formation control involves distributed agents cooperatively achieving their desired formations and destinations. In this case, the control method has to be designed to deal with several problems, such as how to avoid their collisions, how to achieve their desired formation, and how to deal with the actuator limitations. One of the most attractive control schemes to take into account these problems is the use of Distributed Model Predictive Control (DMPC) and an extensive research has already been done, e.g., [11] [12], [13], [16], [19], [20], [21], [27]. This control scheme tries to solve a Finite Horizon Optimal Control Problem (FHOCP) on-line given the current state of the plant and the information of the neighbors to predict their behavior. For example, in [16] the authors consider DMPC for multi-vehicle stabilization for the continuoustime nonlinear systems. In [16], the upper-bound of sampling interval of solving the

\footnotetext{
${ }^{*}$ A condensed conference version has been submitted for conference publication. Kazumune Hashimoto is with the Automatic Control Laboratory, Department of Electrical Engineering, KTH Institute of Technology, 10044 Stockholm, Sweden (e-mail: kazumune@kth.se).

† Shuichi Adachi is with Department of Applied Physics and Physico-Informatics, Keio University, Yokohama, Japan

${ }^{\ddagger}$ Dimos V. Dimarogonas is with the ACCESS Linnaeus Center, School of Electrical Engineering, KTH Royal Institute of Technology, 10044 Stockholm, Sweden (e-mail : dimos@ee.kth.se).
} 
optimal control problem is also derived so that the desired cooperation can be achieved. The readers can also refer to more recent result in [11], where the communication delays are taken into account. For discrete-time nonlinear systems, the reader can refer to [21] for the detailed stability analysis, where the authors apply Input to State Stability (ISS) with respect to neighbors' exchanged information.

One of the most common methods of DMPC is the case of decoupled sub-systems, where each sub-system is not directly influenced by the others. The cost function is instead, coupled and affected by others as a part of the total cost. This implies that choosing the controller is influenced by the cooperation with the neighbors and consequently, may indirectly affect the individual dynamics.

Another attractive research deals with how to reduce energy consumption and usage communication resources in large scale interconnected networks. The battery discharge could be one of the serious concerns due to the communication and computational loads when the battery power is limited but still the control inputs are executed periodically. The event-triggered control schemes have been proposed recently to expand the lifetime of agents by aperiodically executing control inputs, while guaranteeing desired control performances. Event-triggered and self-triggered control are two main different aperiodic control approaches, see, e.g., [5], [6], [7], [9], [24], [25] for the event-based case, and [3], [8], [26] for the self-triggered case. The major difference between event-based and self-triggered control is that, the event-based control scheme requires current measurements of states of the plant, while in the self-triggered case the next control execution is pre-computed without measuring the states of the system based on the prediction and the dynamics.

Although the MPC framework requires to solve the FHOCP periodically, and thus it is clearly computational demanding especially if the dynamics are nonlinear, the application of the event-triggered control to MPC has received some attention in recent years; the readers can refer to [1], [2], [3], [4], [8], [9], [10], [15], [17], [28] for some novel results. In [1], the authors consider deriving event-based MPC for distributed agents having nonlinear dynamics with no additive disturbances, and the FHOCP is aperiodically solved according to an event-based triggering rule with respect to the information error from the neighboring agents based on stability analysis. Furthermore, this is extended to deriving self-triggered conditions in [4]. While these methods may lead to the reduction of the energy consumption, the triggering rules could be conservative as the size of the network increases, since the information error for all of the agent's neighbors is taken into account for the stability criterion. In [3], a self-triggered MPC for single agent having nonholonomic dynamics is derived, where additive bounded disturbances are dealt with. The triggering rule is derived according to robust stability criterion, where the total cost to be minimized is regarded as a Lyapunov candidate to show the Input to State Stability (ISS).

In [17], the authors consider a self-triggered MPC for single agent with linear dynamics. A sub-optimal control input is designed, through which the infinite horizon quadratic cost is evaluated. The event-triggered rule described in [17] takes over the fundamental concept of the event-triggered control, since the piece-wise constant control input is applied aperiodically in order to alleviate not only the computational load but also communication load. This is clearly different from other results on eventtriggered MPC in which the control execution are still periodic. An extended result 
has been recently proposed in [8], where certain disturbances (not necessarily Gaussian noise) are additionally taken into account and the effectiveness of the method is verified through numerical simulations. The reader can also refer to [9] for the case of event-triggered MPC, where the authors derive the triggering condition for a discretetime linear system with additive bounded disturbances. In [28], the authors propose and event-based DMPC for continuous-time nonlinear systems. The key idea is to use a Lyapunov-based MPC, in which the stability property holds in a sample-and-hold fashion by using an additional constraint regarding the optimal cost. A more recent result is proposed in [10], where an event-based condition for single agent with nonlinear continuous time system with additive bounded disturbances is proposed. In [10], the lower bound of inter-execution times is also given, and the feasibility is guaranteed by imposing additional constraints regarding state variables and the prediction horizon.

In this paper, we consider deriving event-based and self-triggered MPC for the cooperation of distributed agents having nonlinear discrete time dynamics, where the FHOCP is aperiodically solved according to certain triggering rules. A main novelty with respect to earlier approaches is that we derive a triggering condition which does not involve the neighbors' information by changing the expression of the Lyapunov function candidate, and thus the periodic usage of communication is not needed even in the event-based case. Moreover, additive bounded disturbances are taken into account, and the corresponding triggering rule is obtained by robust stability of MPC. Therefore, our proposal could lead not only to a reduction of the computational load of MPC but also to the relaxation of over-usage of communication resources for the cooperation of perturbed multi-agent systems. On the other hand, we impose an additional constraint for guaranteeing the stability property. The triggering rule will be obtained for the event-based case at first, which will then be extended for achieving a self-triggered condition by making use of the boundedness of uncertainties between predictive and actual state. The feasibility and stability analysis are also provided.

The rest of this paper is organized as follows. In Section 2, the mathematical modeling and the problem formulation of MPC for distributed agents are described along with several assumptions. In Section 3, an event-based condition is obtained by using robust stability criterion, and then a feasibility analysis is conducted. In Section 4, a self-triggered condition is derived by changing the expression of admissible control inputs, and the corresponding feasibility is also shown by imposing more restrictions on the size of the disturbance. Simulation results verify our proposal in Section 5 , and a summary of the results of this paper is given in Section 6 .

\section{Problem Formulation}

In this section the problem formulation is going to be presented. At first, the mathematical modeling is provided and then the design for MPC for each agent is formulated.

\subsection{Modeling}

We consider a distributed system consisting of $M$ agents, where each agent is controlled by a local Model Predictive Controller. The nominal model for each agent is 
given by a nonlinear difference equation

$$
\hat{x}_{i}(k+1)=f_{i}\left(\hat{x}_{i}(k), u_{i}(k)\right)
$$

where for each $i=1, \ldots, M, \hat{x}_{i}(k) \in \mathbb{R}^{n}$ is the state of $i$-th agent at time $k$ and $u_{i}(k) \in \mathbb{R}^{m}$ is control variable. We further consider that the agent moves under the influence of a certain disturbance. That is, the dynamics of the actual state is given by following perturbed model

$$
x_{i}(k+1)=f_{i}\left(x_{i}(k), u_{i}(k)\right)+w_{i}(k), w_{i}(k) \in \mathcal{W}
$$

where $w_{i}$ is an additive disturbance which belongs to a compact set $w_{i}(k) \in W$ and is assumed to be bounded by $\left\|w_{i}(k)\right\| \leq \bar{w}$, where $\|\cdot\|$ denotes the Euclidean norm. We assume $f_{i}(0,0)=0$ and the constraints for the state and control input on each agent are of the form $x_{i}(k) \in \mathcal{X}_{i}, u_{i}(k) \in \mathcal{U}_{i}$.

\subsection{Distributed MPC Formulation}

In the proposed distributed MPC framework, each agent solves a Finite Horizon Optimal Control Problem (FHOCP) involving the predictive states $\hat{x}_{i}(k+l \mid k)$ and predictive control inputs $u_{i}(k+l \mid k)$ based on its current state, and the information from its neighbors. The current and predictive states and control inputs are denoted in vector format as

$$
\boldsymbol{x}_{i}(k)=\left\{\hat{x}_{i}(k+l \mid k)\right\}_{l=0}^{N}, \quad \boldsymbol{u}_{i}(k)=\left\{u_{i}(k+l \mid k)\right\}_{l=0}^{N-1}
$$

with $\hat{x}_{i}(k \mid k)=x_{i}(k)$. Furthermore, we consider a partially connected structure, where each agent $\mathcal{A}_{i}$ can exchange the state information with neighboring cooperating agents $\mathcal{G}_{i}=\left\{\mathcal{A}_{j}, j \in G_{i}\right\}$, and $G_{i}$ denotes the set of indices of agents belonging to the set $\mathcal{G}_{i}$. The information vector from the neighboring agent $j$ is denoted as $\boldsymbol{z}_{j}(k)=$ $\left\{z_{j}(k+l)\right\}_{l=0}^{N}$, where each component $z_{j}(k+l)$ is the predictive state of the $j$-th agent at $k+l$. This information vector depends on whether event-based or self-triggered control is used, as well as whether the FHOCP is solved, so more specific definitions of the information vector are going to be provided in Sections 3 and 4 .

The overall information which the $i$-th agent obtains from all of its neighboring agents at time $k$ are collected as one stack vector $\boldsymbol{z}_{-i}(k)$ given by

$$
\boldsymbol{z}_{-i}(k)=\operatorname{col}\left(\boldsymbol{z}_{j}(k), j \in G_{i}\right)
$$

Given the state $x_{i}(k)$ at time $k$ and its neighbors' information vector $\boldsymbol{z}_{-i}(k)$, the overall cost function for each agent is given by

$$
\begin{aligned}
& J_{i}\left(\boldsymbol{x}_{i}(k), \boldsymbol{u}_{i}(k), \boldsymbol{z}_{-i}(k)\right) \\
& =J_{i}^{H}\left(\boldsymbol{x}_{i}(k), \boldsymbol{u}_{i}(k)\right)+J_{i}^{Q}\left(\boldsymbol{x}_{i}(k), \boldsymbol{z}_{-i}(k)\right)
\end{aligned}
$$

We will also use the notation $J_{i}(k)=J_{i}^{H}(k)+J_{i}^{Q}(k)$. The cost consists of two terms $: J_{i}^{H}(k)$ is the cost for the agent itself and is given by

$$
\begin{aligned}
J_{i}^{H}(k)= & \sum_{l=0}^{N-1}\left\{h_{i}\left(\hat{x}_{i}(k+l \mid k), u_{i}(k+l \mid k)\right)\right\} \\
& +V_{i}\left(\hat{x}_{i}(k+N \mid k)\right)
\end{aligned}
$$


where $\hat{x}_{i}(k+l \mid k)$ is the predictive state obtained from the nominal model (1), $V_{i}\left(\hat{x}_{i}(k+\right.$ $N \mid k))$ is a terminal cost, and $N$ is the prediction horizon. The second cost $J_{i}^{Q}(k)$ involves the information from the neighbors and is given by

$$
J_{i}^{Q}(k)=\sum_{l=0}^{N-1} \sum_{j \in G^{i}} q_{i j}\left(\hat{x}_{i}(k+l \mid k), z_{j}(k+l)\right)
$$

where $q_{i j}$ is a coupling cost between agent $i$ and $j$. In this paper we assume that the transition cost $h_{i}$ and coupling cost $q_{i j}$ are both given by quadratic functions: $h_{i}\left(x_{i}, u_{i}\right)=\left\|x_{i}\right\|_{F}^{2}+\left\|u_{i}\right\|_{R}^{2}, q_{i j}\left(x_{i}, z_{j}\right)=\left\|x_{i}-z_{j}+d_{i j}\right\|_{Q_{i j}}^{2}$, where the matrices $F, R, Q_{i j}$ are positive definite, and $d_{i j}$ is a desired distance vector between agents $i$ and $j$.

The FHOCP for the perturbed model (2) is now ready to be formulated as follows :

$$
\begin{array}{ll} 
& \min J_{i}\left(\boldsymbol{x}_{i}(k), \boldsymbol{u}_{i}(k), \boldsymbol{z}_{-i}(k)\right) \\
& \boldsymbol{u}_{i}(k) \\
\text { s.t. } & \hat{x}_{i}(k+l+1 \mid k)=f_{i}\left(\hat{x}_{i}(k+l \mid k), u_{i}(k+l \mid k)\right) \\
& \hat{x}_{i}(k+l \mid k) \in \mathcal{X}_{i}^{l} \\
& u_{i}(k+l \mid k) \in U_{i} \\
& \hat{x}_{i}(k+N \mid k) \in \mathcal{X}_{f_{i}} \\
& J_{i}^{H}\left(\boldsymbol{x}_{i}(k), \boldsymbol{u}_{i}(k)\right) \leq \gamma_{i}(k)
\end{array}
$$

where the constraint for $\hat{x}_{i}(k+l \mid k)$ is narrowed to $\hat{x}_{i}(k+l \mid k) \in \mathcal{X}_{i}^{l} \subseteq \mathcal{X}_{i}$ to make sure that there is a robust positively invariant set for the closed loop system where a solution to the FHOCP exists, see [14]. More specifically, the restricted constraint set $\mathcal{X}_{i}^{l}$ is given by $\mathcal{X}_{i}^{l}=\mathcal{X}_{i} \sim B_{i}^{l}$, where $B_{i}^{l}=\left\{x \in \mathbb{R}^{n}:\|x\| \leq \frac{L_{f_{i}}^{l}-1}{L_{f_{i}}-1}\right\}$ and $\sim$ denotes Pontryagin difference.

The terminal constraint $\mathcal{X}_{f_{i}}$ is a set given by $\mathcal{X}_{f_{i}}=\left\{x \in \mathbb{R}^{n}: V_{i}(x) \in \alpha_{v_{i}}\right\}$ and $\mathcal{X}_{f_{i}} \subseteq \mathcal{X}_{i}$. The last constraint $J_{i}^{H}\left(\boldsymbol{x}_{i}(k), \boldsymbol{u}_{i}(k)\right) \leq \gamma_{i}(k)$ is imposed for ensuring the stability for each agent. A more specific definition of $\gamma_{i}(k)$ is formulated later in this text. We make following assumptions for the stability analysis :

Assumption 1. $f_{i}(x, u)$ is Lipschitz continuous with Lipschitz constant $L_{f_{i}}$, i.e.

$$
\left\|f_{i}\left(x_{1}, u\right)-f_{i}\left(x_{2}, u\right)\right\| \leq L_{f_{i}}\left\|x_{1}-x_{2}\right\|
$$

Assumption 2. The running costs $h_{i}\left(x_{i}, u_{i}\right)$ is Lipschitz continuous in $x_{i}$, with Lipschitz constant $L_{h_{i}}$.

Assumption 3. There exists a local stabilizing controller $\kappa_{i}(x) \in U_{i}$ in the sense that

$$
V_{i}\left(f_{i}\left(x, \kappa_{i}(x)\right)\right)-V_{i}(x) \leq-h_{i}\left(x, \kappa_{i}(x)\right)
$$

for all $x \in \Phi_{i}$, where $\Phi_{i}$ is a compact set given by $\Phi_{i}=\left\{x \in \mathbb{R}^{n}: V_{i}(x) \leq \alpha_{i}\right\}$ and $\Phi_{i} \subseteq \mathcal{X}_{i}^{N-m}$ for $m=1, \cdots, N-1$. 
Assumption 4. The terminal cost $V_{i}(x)$ is Lipschitz in $x \in \Phi_{i}$ with Lipschitz constant $L_{V_{i}}$.

Assumption 5. The set $\mathcal{X}_{f_{i}}=\left\{x \in \mathbb{R}^{n}: V_{i}(x) \in \alpha_{v_{i}}\right\}$ is such that for all $x \in \Phi_{i}$, $f\left(x, \kappa_{i}(x)\right) \in \mathcal{X}_{f_{i}} \subseteq \Phi_{i}$.

Remark 1. All of these assumptions are fairly standard for guaranteeing ISS under additive bounded disturbances; see e.g., [14], [23]. Note that the set $\mathcal{X}_{i}^{l}$ could be very small if the Lipschitz constant $L_{f_{i}}$ is relatively large, especially when it is larger than 1. This problem is also remarked in [14], where several methods to reduce this conservativeness are given. For example, the control parametrization method can be used by giving a feedback structure $u_{i}(k)=K x_{i}(k)+v_{i}(k)$ to reduce $L_{f_{i}}$, where $v_{i}(k)$ is a new control variable.

The solution to the FHOCP gives an optimal control input sequence and the corresponding predictive states denoted by

$$
\boldsymbol{x}_{i}^{*}(k)=\left\{\hat{x}_{i}^{*}(k+l \mid k)\right\}_{l=0}^{N}, \quad \boldsymbol{u}_{i}^{*}(k)=\left\{u_{i}^{*}(k+l \mid k)\right\}_{l=0}^{N-1}
$$

where $\hat{x}_{i}^{*}(k \mid k)=x_{i}(k)$. Then, in the event-triggered formulation, some part of this optimal input is applied to the system, i.e.,

$$
u_{i}^{a p}(k+l \mid k)=u_{i}^{*}(k+l \mid k), \quad l=0, \cdots, m-1
$$

where $k+m$ denotes the next triggering time, which is obtained by the triggering condition. Before deriving the triggering condition, some useful Lemmas are given by following :

Lemma 1. The difference between the true state $x_{i}(k+l)$ and the predictive state $\hat{x}_{i}(k+l \mid k)$ is bounded by $\left\|\hat{x}_{i}(k+l \mid k)-x_{i}(k+l)\right\| \leq \delta_{i}(l)$ where $\delta_{i}(l)=\frac{L_{f_{i}}^{l}-1}{L_{f_{i}}-1} \bar{w}$.

For the proof the reader can refer to [14]. The following is an extension of Lemma 2 in [14].

Lemma 2. Let $\mathcal{X}_{i}^{l}$ be given by $\mathcal{X}_{i}^{l}=\mathcal{X}_{i} \sim B_{i}^{l}$, where $B_{i}^{l}=\left\{x \in R^{n}:\|x\| \leq \frac{L_{f_{i}}^{l}-1}{L_{f_{i}}-1}\right\}$ for $l \geq 1$, and let $x \in \mathcal{X}_{i}^{l}$ and $y \in \mathbb{R}^{n}$ be such that $\|x-y\| \leq L_{f_{i}}^{l-m} \frac{L_{f_{i}}^{m}-1}{L_{f_{i}}-1} \bar{w}$ for $0 \leq m<l$. Then $y \in \mathcal{X}_{i}^{l-m}$.

Proof. Let $z=y-x+e_{l-m}$, where $e_{l-m} \in B_{i}^{l-m}$ and $x \in \mathcal{X}_{i}^{l}$. We get

$$
\begin{aligned}
\|z\| & \leq\|x-y\|+\left\|e_{l-m}\right\| \\
& =L_{f_{i}}^{l-m} \frac{L_{f_{i}}^{m}-1}{L_{f_{i}}-1} \bar{w}+\frac{L_{f_{i}}^{l-m}-1}{L_{f_{i}}-1} \bar{w}=\frac{L_{f_{i}}^{l}-1}{L_{f_{i}}-1} \bar{w}
\end{aligned}
$$

Hence $z \in B_{i}^{l}$. Since $y+e_{l-m}=z+x \in \mathcal{X}_{i}$, we obtain $y \in \mathcal{X}_{i}^{l-m}$. 


\section{Event-based MPC}

\subsection{Deriving the event-based condition}

In this section the event-based triggering rules are derived. Assuming that we solved the FHOCP at $k$, then this provides an optimal control sequence $\boldsymbol{u}_{i}^{*}(k)$ and the corresponding optimal cost denoted by $J_{i}^{*}(k)=J_{i}^{H *}(k)+J_{i}^{Q *}(k)$, where $J_{i}^{H *}(k)=$ $J_{i}^{H}\left(\boldsymbol{x}_{i}^{*}(k), \boldsymbol{u}_{i}^{*}(k)\right)$ and $J_{i}^{Q *}(k)=J_{i}^{Q}\left(\boldsymbol{x}_{i}^{*}(k), \boldsymbol{z}_{-i}(k)\right)$. Then, consider that the following control sequence $\overline{\boldsymbol{u}}_{i}(k+m)=\left\{\bar{u}_{i}(k+l \mid k+m)\right\}_{l=m}^{m+N-1}$ where $1 \leq m<N$ is used to obtain the predictive state sequence $\overline{\boldsymbol{x}}_{i}(k+m)=\left\{\bar{x}_{i}(k+l \mid k+m)\right\}_{l=m}^{m+N}$ (given $\bar{x}_{i}(k+m \mid k+m)=x_{i}(k+m)$ ) from $k+m$ : for $m=1$,

$$
\begin{aligned}
& \bar{u}_{i}(k+l \mid k+1)= \\
& \left\{\begin{array}{l}
u_{i}^{*}(k+l \mid k)(\text { for } l=1, \cdots, N-1) \\
\kappa_{i}(\bar{x}(k+l \mid k+1))(\text { for } l=N)
\end{array}\right.
\end{aligned}
$$

For $1<m<N$,

$$
\begin{aligned}
& \bar{u}_{i}(k+l \mid k+m)= \\
& \left\{\begin{array}{l}
\bar{u}_{i}(k+l \mid k+m-1)(\text { for } l=m, \cdots, N+m-2) \\
\kappa_{i}(\bar{x}(k+l \mid k+m))(\text { for } l=N+m-1)
\end{array}\right.
\end{aligned}
$$

Instead of solving the FHOCP at $k+m$, we will assume that this admissible control input $\overline{\boldsymbol{u}}_{i}(k+m)$ is going to be applied to check if the stability is still guaranteed. The corresponding cost is simply denoted as $\bar{J}_{i}(k+m)=\bar{J}_{i}^{H}(k+m)+\bar{J}_{i}^{Q}(k+m)$, where $\bar{J}_{i}^{H}(k+m)=J_{i}^{H}\left(\overline{\boldsymbol{x}}(k+m), \overline{\boldsymbol{u}}_{i}(k+m)\right)$ and $\bar{J}_{i}^{Q}(k+m)=J_{i}^{Q}\left(\overline{\boldsymbol{x}}(k+m), \boldsymbol{z}_{-i}(k+m)\right)$.

Now we propose the following triggering rule.

(Triggering rule): The FHOCP is triggered when $J_{i}^{H}(k)$ is not guaranteed to decrease.

This means that we take $J_{i}^{H}(k)=J_{i}^{H}\left(\boldsymbol{x}_{i}(k), \boldsymbol{u}_{i}(k)\right)$ as a Lyapunov candidate, instead of using the total cost $J_{i}(k)$. The reason for this is that if we had the total cost as a Lyapunov candidate, we would need to take into account the information $\boldsymbol{z}_{-i}(k)$ to evaluate triggering conditions and thus the periodic usage of communication resources would be required. Furthermore, the uncertainties of this information vector would be considered for all the neighbors, and thus the triggering condition might have been more conservative. Thus by taking only $J_{i}^{H}(k)$ as a Lyapunov candidate, which involves only the information of the agent $i$ itself, the reduction of not only the utilization of communication resources but also of the conservativeness of the triggering rule can be achieved.

The problem when the partial cost is used here is, however, that the optimal control inputs are obtained by evaluating the total cost $J_{i}(k)$ and thus $\bar{J}_{i}^{H}(k)$ does not necessarily follow $J_{i}^{H *}(k) \leq \bar{J}_{i}^{H}(k)$, and the stability conditions are hard to verify. Motivated by this, we impose here an additional constraint for $J_{i}^{H}(k)$ in $J_{i}^{H}\left(\boldsymbol{x}_{i}(k), \boldsymbol{u}_{i}(k)\right) \leq$ $\gamma_{i}(k)$, which corresponds to $J_{i}^{H}\left(\boldsymbol{x}_{i}(k+m), \boldsymbol{u}_{i}(k+m)\right) \leq \gamma_{i}(k+m)$ when the FHOCP is triggered at $k+m$. The upper bound $\gamma_{i}(k+m)$ is defined as

$$
\begin{aligned}
\gamma_{i}(k+m)= & \bar{J}_{i}^{H}(k+m-1)+L_{p i} \cdot \bar{w} \\
& -h_{i}\left(x_{i}(k+m-1), u_{i}^{*}(k+m-1 \mid k)\right)
\end{aligned}
$$


where $L_{p i}=L_{h_{i}} \frac{L_{f_{i}}^{N-1}-1}{L_{f_{i}}-1}+L_{V_{i}} L_{f_{i}}^{N-1}$, and $\bar{J}_{i}^{H}(k+m-1)$ is replaced with the optimal cost $J_{i}^{H *}(k)$ when $m=1$.

Having obtained $J_{i}^{H *}(k)$ at $k$, we first check if this cost is guaranteed to decrease from $k$ to $k+1$. Regarding this point, consider that an admissible control input $\overline{\boldsymbol{u}}_{i}(k+1)$ is used for $k+1$ to obtain $\bar{J}_{i}^{H}(k+1)$. If we show that $\bar{J}_{i}^{H}(k+1)<J_{i}^{H *}(k)$, the stability is already guaranteed without having to solve the FHOCP. The difference between the next cost $\bar{J}_{i}^{H}(k+1)$ and the optimal cost $J_{i}^{H *}(k)$ is given by

$$
\begin{aligned}
\Delta J_{i 1}^{H}= & \bar{J}_{i}^{H}(k+1)-J_{i}^{H *}(k) \\
& =-h_{i}\left(x_{i}(k), u_{i}^{*}(k \mid k)\right) \\
& +\sum_{l=1}^{N-1}\left\{h_{i}\left(\bar{x}_{i}(k+l \mid k+1), \bar{u}_{i}(k+l \mid k+1)\right)\right. \\
& \left.-h_{i}\left(\hat{x}_{i}^{*}(k+l \mid k), u_{i}^{*}(k+l \mid k)\right)\right\} \\
& -V_{i}\left(\hat{x}_{i}^{*}(k+N \mid k)\right)+V_{i}\left(\bar{x}_{i}(k+N+1 \mid k+1)\right. \\
& +h_{i}\left(\bar{x}_{i}(k+N \mid k+1), \kappa_{i}(\cdot)\right) \\
& -V_{i}\left(\bar{x}_{i}(k+N \mid k+1)\right)+V_{i}\left(\bar{x}_{i}(k+N \mid k+1)\right)
\end{aligned}
$$

By using the Assumption 4 for terminal cost and state, we get

$$
\begin{aligned}
& V_{i}\left(\bar{x}_{i}(k+N+1 \mid k+1)\right)-V_{i}\left(\bar{x}_{i}(k+N \mid k+1)\right) \\
& \leq-h_{i}\left(\bar{x}_{i}(k+N \mid k+1), \kappa_{i}\left(\bar{x}_{i}(k+N \mid k+1)\right)\right) .
\end{aligned}
$$

Since $\bar{u}_{i}(k+l \mid k+1)=u_{i}^{*}(k+l \mid k)$ for $l=1, \cdots, N-1$, the difference for the transition cost $h_{i}$ in the summation can be bounded by

$$
\begin{aligned}
& \mid h_{i}\left(\bar{x}_{i}(k+l \mid k+1), \bar{u}_{i}(k+l \mid k+1)\right) \\
& -h_{i}\left(\hat{x}_{i}^{*}(k+l \mid k), u_{i}^{*}(k+l \mid k)\right) \mid \\
& \leq L_{h_{i}} L_{f_{i}}^{l-1} \bar{w}
\end{aligned}
$$

Furthermore, the difference for the terminal cost $V_{i}$ is bounded by

$$
\begin{aligned}
& \left|V_{i}\left(\hat{x}_{i}^{*}(k+N \mid k)\right)-V_{i}\left(\bar{x}_{i}(k+N \mid k+1)\right)\right| \\
& \leq L_{V_{i}} L_{f_{i}}^{N-1} \bar{w}
\end{aligned}
$$

Therefore, the bound is given by

$$
\begin{aligned}
\Delta J_{i 1}^{H} & \leq-h_{i}\left(x_{i}(k), u_{i}^{*}(k \mid k)\right) \\
& +\sum_{l=1}^{N-1}\left(L_{h_{i}} L_{f_{i}}^{l-1} \bar{w}\right)+L_{V_{i}} L_{f_{i}}^{N-1} \bar{w} \\
& \leq-h_{i}\left(x_{i}(k), u_{i}^{*}(k \mid k)\right)+L_{p i} \cdot \bar{w}
\end{aligned}
$$

Letting

$$
L_{p i} \bar{w} \leq \sigma \cdot h_{i}\left(x_{i}(k), u_{i}^{*}(k \mid k)\right)
$$

where $0<\sigma<1$, we obtain

$$
\Delta J_{i 1}^{H} \leq(\sigma-1) \cdot h_{i}\left(x_{i}(k), u_{i}^{*}(k \mid k)\right)<0
$$

and so the convergence property is guaranteed. Thus if (13) holds, the FHOCP is determined not to be solved at $k+1$. Now assume that the triggering condition (13) 
is satisfied and we obtain $\bar{J}_{i}^{H}(k+1)$ at $k+1$. Then, we further consider that the admissible control input $\overline{\boldsymbol{u}}(k+2)$ is going to be used for $k+2$ to get the next cost $\bar{J}_{i}^{H}(k+2)$, and take a difference from $\bar{J}_{i}^{H}(k+1)$ similarly to (9), i.e.,

$$
\begin{aligned}
\Delta J_{i 2}^{H} & =\bar{J}_{i}^{H}(k+2)-\bar{J}_{i}^{H}(k+1) \\
& =-h_{i}\left(x_{i}(k+1), u_{i}^{*}(k+1 \mid k)\right) \\
& +\sum_{l=2}^{N}\left\{h_{i}\left(\bar{x}_{i}(k+l \mid k+2), \bar{u}_{i}(k+l \mid k+2)\right)\right. \\
& \left.-h_{i}\left(\bar{x}_{i}(k+l \mid k+1), \bar{u}_{i}(k+l \mid k+1)\right)\right\} \\
& -V_{i}\left(\bar{x}_{i}(k+N+1 \mid k+1)\right) \\
& +V_{i}\left(\bar{x}_{i}(k+N+2 \mid k+2)\right. \\
& +h_{i}\left(\bar{x}_{i}(k+N+1 \mid k+2), \kappa_{i}(\cdot)\right) \\
& -V_{i}\left(\bar{x}_{i}(k+N+1 \mid k+2)\right) \\
& +V_{i}\left(\bar{x}_{i}(k+N+1 \mid k+2)\right)
\end{aligned}
$$

From (6), $\bar{u}_{i}(k+l \mid k+1)=\bar{u}(k+l \mid k+2)$ for $l=2, \cdots, N$. By using the Assumption 4 for the terminal cost, we get

$$
\begin{aligned}
& V_{i}\left(\bar{x}_{i}(k+N+2 \mid k+2)\right)-V_{i}\left(\bar{x}_{i}(k+N+1 \mid k+2)\right) \\
& \leq-h_{i}\left(\bar{x}_{i}(k+N+1 \mid k+2), \kappa_{i}\left(\bar{x}_{i}(k+N+1 \mid k+2)\right)\right)
\end{aligned}
$$

From Lemma 1, the difference between the transition $\operatorname{costs} h_{i}$ in the summation above is bounded by

$$
\begin{aligned}
& \mid h_{i}\left(\bar{x}_{i}(k+l \mid k+2), \bar{u}_{i}(k+l \mid k+2)\right) \\
& -h_{i}\left(\bar{x}_{i}(k+l \mid k+1), \bar{u}_{i}(k+l \mid k+1)\right) \mid \\
& \leq L_{h_{i}} L_{f_{i}}^{l-2} \bar{w}
\end{aligned}
$$

Furthermore, the the difference for the terminal $\operatorname{cost} V_{i}$ is bounded by

$$
\begin{aligned}
& \left|V_{i}\left(\bar{x}_{i}(k+N+1 \mid k+2)\right)-V_{i}\left(\bar{x}_{i}(k+N+1 \mid k+1)\right)\right| \\
& \leq L_{V_{i}} L_{f_{i}}^{N-1} \bar{w}
\end{aligned}
$$

which is also the same bound as in (11). Therefore, we get

$$
\Delta J_{i 2}^{H} \leq-h_{i}\left(x_{i}(k+1), u_{i}^{*}(k+1 \mid k)\right)+L_{p i} \cdot \bar{w}
$$

Therefore, by letting $L_{p i} \bar{w} \leq \sigma \cdot h_{i}\left(x_{i}(k+1), u_{i}^{*}(k+1 \mid k)\right)$, we get $\Delta J_{i 2}^{H}<0$, so the convergence property is guaranteed. Thus if this condition is satisfied, the FHOCP is determined not to be solved at $k+2$. By using the same procedure, we get the bound of $\Delta J_{i m}^{H}=\bar{J}_{i}^{H}(k+m)-\bar{J}_{i}^{H}(k+m-1)$ for $1 \leq m<N$ as

$$
\begin{aligned}
\Delta J_{i m}^{H}= & \bar{J}_{i}^{H}(k+m)-\bar{J}_{i}^{H}(k+m-1) \\
\leq & -h_{i}\left(x_{i}(k+m-1), u_{i}^{*}(k+m-1 \mid k)\right) \\
& +L_{p i} \cdot \bar{w}
\end{aligned}
$$

and so the triggering rule is given by

$$
L_{p i} \cdot \bar{w} \leq \sigma \cdot h_{i}\left(x_{i}(k+m-1), u_{i}^{*}(k+m-1 \mid k)\right) .
$$

In this way, the FHOCP is aperiodically solved while convergence is preserved. Therefore, the event-based MPC is formulated as follows 
(Event-based MPC): Assume that the FHOCP is solved at $k$. For $k+m$ where $1 \leq$ $m<N$, the FHOCP is triggered at $k+m$ when (18) is violated. When (18) is satisfied for all $1 \leq m<N$, then the FHOCP is triggered at $k+N$.

Remark 2. Note that the triggering rule does not involve any information from $i$ 's neighbors, and thus there is no need of communication when the FHOCP is not solved. Also note that this triggering rule is categorized as event-based control, since at each time we need to observe the true state to calculate not only the transition cost $h_{i}$ but also $\gamma_{i}(k+m)$; this is because, in order to calculate $\gamma_{i}(k+m), \overline{\boldsymbol{u}}(k+m-1)$ is based on $\overline{\boldsymbol{u}}(k+m-2)$, which involves the local controller $\kappa_{i}(\bar{x}(k+N+m-3 \mid k+m-2)$ which in turn needs to be computed from the actual $x_{i}(k+m-2)$. This results in needing the measurements $x_{i}(k+m-3), \cdots, x_{i}(k+1)$.

Definition 1 (Information vector for event-based case). Let $\boldsymbol{z}_{i}(k+1)$ be the information vector that should be transmitted from agent $i$ at time $k$. This information is sent to its neighbor, e.g., $j$, only when $j$ decides to solve the FHOCP at $k+1$. When the agent $i$ solves the FHOCP at $k$, the following optimal predictive states are transmitted

$$
\boldsymbol{z}_{i}(k+1)=\left\{\hat{x}_{i}^{*}(k+1 \mid k), \cdots, \hat{x}_{i}^{*}(k+N \mid k), f_{i}\left(\hat{x}_{i}^{*}(k+N \mid k), \kappa_{i}(\cdot)\right)\right\} .
$$

When it is not solved, the predictive states obtained from $\overline{\boldsymbol{u}}(k)$ are transmitted, i.e.,

$$
\boldsymbol{z}_{i}(k+1)=\left\{\bar{x}_{i}(k+1 \mid k), \cdots, \bar{x}_{i}(k+N \mid k), f_{i}\left(\bar{x}_{i}(k+N \mid k), \kappa_{i}(\cdot)\right)\right\} .
$$

\subsection{Feasibility analysis}

In this section the feasibility analysis of the FHOCP for each agent is going to be given. Analyzing the feasibility is important in the MPC framework in order to ensure that there exists a solution satisfying all the constraints when the FHOCP is triggered. In the event-triggered formulation, it is going to be shown that if we solve the FHOCP at $k$, the FHOCP is feasible whenever until prediction horizon it is again going to be solved in the future $k+m$. This is different from the standard (periodic) MPC where we solve the FHOCP every time, although the feasibility can be shown in a similar manner to [14] by restricting the size of the disturbance.

The main theorem for the feasibility is provided below.

Theorem 1. Let the system be described by (2), and assume that all Assumptions 15 are satisfied. Then, the FHOCP solved by agent $i$ is feasible if the disturbance is bounded by

$$
\bar{w} \leq \frac{\left(\alpha_{i}-\alpha_{v_{i}}\right)}{L_{V_{i}} L_{f_{i}}^{N-1}}
$$

for all $i=1, \cdots, M$.

Proof. Assume that we successfully solve FHOCP at time $k$ to get the optimal input sequence $\boldsymbol{u}_{i}^{*}(k)$ and the corresponding optimal cost $J_{i}^{*}(k)$, and then from the triggering rule, the next FHOCP is determined to be solved at $k+m$ where $1 \leq m<N$. The FHOCP is shown to be feasible at $k+m$ that there exists a solution satisfying all the constraints in (3) by considering that $\overline{\boldsymbol{u}}_{i}(k+m)$ given by either (6) or (7) is applied. 
1. $\overline{\boldsymbol{u}}_{i}(k+m) \in U_{i}$

This is clearly admissible from the expression of $\bar{u}_{i}(k+l \mid k+m)$ given by (6) and (7).

2. $\bar{x}_{i}(k+l \mid k+m) \in \mathcal{X}_{i}^{l-m}$ for $l=m+1, \cdots, N+m-1$.

First we show this for $m=1$. Since $\bar{u}_{i}(k+l \mid k+1)=u_{i}^{*}(k+l \mid k)$, we first obtain

$$
\left\|\bar{x}_{i}(k+l \mid k+1)-\hat{x}_{i}^{*}(k+l \mid k)\right\| \leq L_{f_{i}}^{l-1} \bar{w}
$$

where $\hat{x}_{i}^{*}(k+l \mid k) \in \mathcal{X}_{i}^{l}$. Thus from Lemma 2 (for $\left.m=1\right), \bar{x}_{i}(k+l \mid k+1) \in$ $\mathcal{X}_{i}^{l-1}$ for $l=2, \cdots, N$. For $m=2$, since $\bar{u}_{i}(k+l \mid k+2)=\bar{u}_{i}(k+l \mid k+1)$ for $l=2, \cdots, N$, we get

$$
\left\|\bar{x}_{i}(k+l \mid k+2)-\bar{x}_{i}(k+l \mid k+1)\right\| \leq L_{f_{i}}^{l-2} \bar{w}
$$

for $l=3, \cdots, N+1$, where $\bar{x}_{i}(k+l \mid k+1) \in \mathcal{X}_{i}^{l-1}$. Thus from Lemma 2 (for $m=1), \bar{x}_{i}(k+l \mid k+2) \in \mathcal{X}_{i}^{l-2}$ for $l=3, \cdots, N+1$. By recursion, we get $\bar{x}_{i}(k+l \mid k+m) \in \mathcal{X}_{i}^{l-m}$ for $l=m+1, \cdots, N+m-1$.

3. $\bar{x}_{i}(k+m+N \mid k+m) \in \mathcal{X}_{f_{i}}$

First we show $\bar{x}_{i}(k+m+N-1 \mid k+m) \in \Phi_{i}$. For $m=1$, by using

$$
\left\|\bar{x}_{i}(k+N \mid k+1)-\hat{x}_{i}^{*}(k+N \mid k)\right\| \leq L_{f_{i}}^{N-1} \bar{w}
$$

we get that

$$
V_{i}\left(\bar{x}_{i}(k+N \mid k+1)\right) \leq \alpha_{v_{i}}+L_{V_{i}} L_{f_{i}}^{N-1} \bar{w} \leq \alpha_{i}
$$

Hence $\bar{x}_{i}(k+N \mid k+1) \in \Phi_{i}$, thus by using Assumption 5, we obtain $\bar{x}_{i}(k+$ $N+1 \mid k+1) \in \mathcal{X}_{f_{i}}$. Similarly as above, for $m=2$,

$$
\begin{aligned}
& \| \bar{x}_{i}(k+N+1 \mid k+2) \\
& -\bar{x}_{i}(k+N+1 \mid k+1) \| \leq L_{f_{i}}^{N-1} \bar{w}
\end{aligned}
$$

and we get

$$
V_{i}\left(\bar{x}_{i}(k+N+1 \mid k+2)\right) \leq \alpha_{v_{i}}+L_{V_{i}} L_{f_{i}}^{N-1} \bar{w} \leq \alpha_{i}
$$

Hence $\bar{x}_{i}(k+N+1 \mid k+2) \in \Phi_{i}$, thus by using Assumption 5, we get $\bar{x}_{i}(k+N+$ $2 \mid k+2) \in \mathcal{X}_{f_{i}}$. Therefore we recursively get that $\bar{x}_{i}(k+m+N-1 \mid k+m) \in \Phi_{i}$ and $\bar{x}_{i}(k+m+N \mid k+m) \in \mathcal{X}_{f_{i}}$.

4. $\bar{J}_{i}^{H}(k+m) \leq \gamma_{i}(k+m)$

First we check for $m=1$. The difference between $\bar{J}_{i}^{H}(k+1)$ and the optimal cost $J_{i}^{H *}(k)$ is bounded according to (12), and thus we get

$$
\begin{aligned}
\bar{J}_{i}^{H}(k+1) & \leq J_{i}^{H *}(k)-h_{i}\left(x_{i}(k), u_{i}^{*}(k \mid k)\right)+L_{p i} \cdot \bar{w} \\
& =\gamma_{i}(k+1)
\end{aligned}
$$


Thus, $\bar{J}_{i}^{H}(k+1)$ satisfies $\bar{J}_{i}^{H}(k+1) \leq \gamma_{i}(k+1)$ and so the feasibility for this constraint is guaranteed when $m=1$. For $1<m<N$, we obtain from (17) that

$$
\begin{aligned}
\bar{J}_{i}^{H}(k+m) & \leq \bar{J}_{i}^{H}(k+m-1)+L_{p i} \cdot \bar{w} \\
& -h_{i}\left(x_{i}(k+m-1), u_{i}^{*}(k+m-1 \mid k)\right) \\
& =\gamma_{i}(k+m)
\end{aligned}
$$

Therefore, the cost at step $k+m$ satisfies $\bar{J}_{i}^{H}(k+m) \leq \gamma_{i}(k+m)$, so the feasibility for the last constraint is guaranteed for $m=1, \cdots, N-1$. This completes the proof for the feasibility.

\section{Self-triggered MPC}

In this section the self-triggered MPC is formulated. The self-triggered condition is derived by modifying $\overline{\boldsymbol{u}}(k)$ in order to calculate $\gamma_{i}(k)$ without needing measurement of the states at time instants, while we impose an additional condition for guaranteeing the feasibility. Some further assumptions to derive the self-triggered condition are in order :

Assumption 6. The nonlinear function with local controller $f_{i}\left(x_{i}, \kappa_{i}\left(x_{i}\right)\right)$ is Lipschitz continuous with Lipschitz constant $L_{F \kappa_{i}}$ in $x_{i} \in \Phi_{i}$.

Assumption 7. The running cost with local controller $h_{i}\left(x_{i}, \kappa_{i}\left(x_{i}\right)\right)$ is Lipschitz continuous in $x_{i}$, with Lipschitz constant $L_{H \kappa_{i}}$ in $x_{i} \in \Phi_{i}$.

We should note here that these are not strict assumptions to obtain $L_{F \kappa_{i}}<\infty$, $L_{H \kappa_{i}}<\infty$, since the state is constrained to be inside the terminal region $x_{i} \in \Phi_{i}$ and these are not defined globally.

\subsection{Deriving the self-triggered condition}

In this section we derive the self-triggered condition. We use the same triggering logic as in the event-based case; we check if $J_{i}^{H}(k)$ as a Lyapunov candidate is decreasing, and the FHOCP is going to be solved when this condition is violated. Similarly to the event-based case, assume that we have solved the FHOCP at $k$, we have an optimal control sequence $\boldsymbol{u}_{i}^{*}(k)$ and the corresponding states $\boldsymbol{x}_{i}^{*}(k)$ and the cost $J_{i}^{H *}(k)$. Then, consider that the following modified control sequence $\overline{\boldsymbol{u}}_{i}(k+m)=\left\{\bar{u}_{i}(k+l \mid k+\right.$ $m)\}_{l=m}^{m+N-1}$ is used to get $\overline{\boldsymbol{x}}_{i}(k+m)=\left\{\bar{x}_{i}(k+l \mid k+m)\right\}_{l=m}^{m+N}$ (given $\bar{x}_{i}(k+m \mid k+$ $\left.m)=x_{i}(k+m)\right)$ from $k+m$, where each component is given by

$$
\begin{aligned}
& \bar{u}_{i}(k+l \mid k+m)= \\
& \left\{\begin{array}{l}
u_{i}^{*}(k+l \mid k)(\text { for } l=m, \cdots, N-1) \\
\kappa_{i}(\bar{x}(k+l \mid k+m))(\text { for } l=N, \cdots, m+N-1)
\end{array}\right.
\end{aligned}
$$

The control inputs are different from those in event-based case since the local controllers are all used after $k+N$. The corresponding cost is again denoted as $\bar{J}_{i}^{H}(k)$, 
and $\gamma_{i}(k)$ is given by (8). Changing the expression of $\overline{\boldsymbol{u}}(k)$ allows us to calculate $\gamma_{i}(k+m)$ without observing the actual states $x_{i}(k+1), \cdots, x_{i}(k+m-2)$, as we only need to measure the most recent state $x_{i}(k+m-1)$ to calculate $\bar{J}_{i}(k+m-1)^{1}$ Furthermore, for notational simplicity in the sequel, we define $L_{S i}(m)$ as

$$
L_{S i}(m)=L_{h_{i}} \frac{L_{f_{i}}^{N-m}-1}{L_{f_{i}}-1}+L_{V_{i}} L_{F \kappa_{i}}^{m-1} L_{f_{i}}^{N-m}+L_{f_{i}}^{N-m} \frac{L_{H \kappa_{i}}^{m}-L_{H \kappa_{i}}}{L_{H \kappa_{i}}-1}
$$

where $L_{S i}(1)=L_{p i}$ if $m=1$.

Now we first check if $\bar{J}_{i}^{H}(k+1)<J_{i}^{H *}(k)$. At $k+1, \overline{\boldsymbol{u}}_{i}(k+1)$ has the same expression as in (6) for the event-based case, so $\Delta J_{i 1}^{H}$ is bounded by

$$
\Delta J_{i 1}^{H} \leq-h_{i}\left(x_{i}(k), u_{i}^{*}(k \mid k)\right)+L_{S i}(1) \cdot \bar{w}
$$

By letting

$$
L_{S i}(1) \cdot \bar{w} \leq \sigma \cdot h_{i}\left(x_{i}(k), u_{i}^{*}(k \mid k)\right)
$$

where $0<\sigma<1$, we obtain $\Delta J_{i 1}^{H}<0$ and so the convergence is guaranteed. This is a self-triggered condition, since the actual state $x_{i}(k)$ is known. Furthermore, as we will see in the next section, the following triggering condition needs to be added in order to guarantee the feasibility.

$$
L_{S i}(2) \leq L_{p i}
$$

This is the extra condition, but it could be less conservative by changing the size of the terminal region so that (25) will dominate as the triggering rule. Thus the FHOCP is solved when either (26) or (25) is violated. The bound of the difference $\Delta J_{i 2}^{H}$ should be considered more carefully as the different local controllers are used from $k+N$;

$$
\begin{aligned}
\Delta J_{i 2}^{H} & =\bar{J}_{i}^{H}(k+2)-\bar{J}_{i}^{H}(k+1) \\
& \leq-h_{i}\left(x_{i}(k+1), u_{i}^{*}(k+1 \mid k)\right) \\
& +\sum_{l=2}^{N-1}\left\{h_{i}\left(\bar{x}_{i}(k+l \mid k+2), \bar{u}_{i}(k+l \mid k+2)\right)\right. \\
& \left.-h_{i}\left(\bar{x}_{i}(k+l \mid k+1), \bar{u}_{i}(k+l \mid k+1)\right)\right\} \\
& +h_{i}\left(\bar{x}_{i}(k+N \mid k+2), \kappa_{i}(\cdot)\right) \\
& -h_{i}\left(\bar{x}_{i}(k+N \mid k+1), \kappa_{i}(\cdot)\right) \\
& -V_{i}\left(\bar{x}_{i}(k+N+1 \mid k+1)\right) \\
& +V_{i}\left(\bar{x}_{i}(k+N+2 \mid k+2)\right. \\
& +h_{i}\left(\bar{x}_{i}(k+N+1 \mid k+2), \kappa_{i}(\cdot)\right) \\
& -V_{i}\left(\bar{x}_{i}(k+N+1 \mid k+2)\right) \\
& +V_{i}\left(\bar{x}_{i}(k+N+1 \mid k+2)\right)
\end{aligned}
$$

By using the Assumption 4 for the terminal cost, we get

$$
\begin{aligned}
& V_{i}\left(\bar{x}_{i}(k+N+2 \mid k+2)\right)-V_{i}\left(\bar{x}_{i}(k+N+1 \mid k+2)\right) \\
& \leq-h_{i}\left(\bar{x}_{i}(k+N+1 \mid k+2), \kappa_{i}\left(\bar{x}_{i}(k+N+1 \mid k+2)\right)\right)
\end{aligned}
$$

\footnotetext{
${ }^{1}$ In the condensed version submitted as a conference paper, this control input is used for both event-based and self-triggered cases in order to allow us to show the feasibility at the same time. However, the allowable size of the disturbances become restrictive for the event-based case.
} 
We also have

$$
\begin{aligned}
& \mid h_{i}\left(\bar{x}_{i}(k+l \mid k+2), \bar{u}_{i}(k+l \mid k+2)\right) \\
& -h_{i}\left(\bar{x}_{i}(k+l \mid k+1), \bar{u}_{i}(k+l \mid k+1)\right) \mid \\
& \leq L_{h_{i}} L_{f_{i}}^{l-2} \bar{w}
\end{aligned}
$$

for $l=2, \cdots, N-1$. The difference for the terminal cost $V_{i}$ is bounded by

$$
\begin{aligned}
& \left|V_{i}\left(\bar{x}_{i}(k+N+1 \mid k+2)\right)-V_{i}\left(\bar{x}_{i}(k+N+1 \mid k+1)\right)\right| \mid \\
& \leq L_{V_{i}}|| f_{i}\left(\bar{x}_{i}(k+N \mid k+2), \kappa_{i}\left(\bar{x}_{i}(k+N \mid k+2)\right)\right) \\
& -f_{i}\left(\bar{x}_{i}(k+N \mid k+1), \kappa_{i}\left(\bar{x}_{i}(k+N \mid k+1)\right)\right)|| \\
& \leq L_{V_{i}} L_{F \kappa_{i}}|| \bar{x}_{i}(k+N \mid k+2)-\bar{x}_{i}(k+N \mid k+1)|| \\
& \leq L_{V_{i}} L_{F \kappa_{i}} L_{f_{i}}^{N-2} \bar{w}
\end{aligned}
$$

The difference between $h_{i}\left(\bar{x}_{i}(k+N \mid k+2), \kappa_{i}(\cdot)\right)$ and $h_{i}\left(\bar{x}_{i}(k+N \mid k+1), \kappa_{i}(\cdot)\right)$ is bounded by

$$
\begin{aligned}
& \mid h_{i}\left(\bar{x}_{i}(k+N \mid k+2), \kappa_{i}\left(\bar{x}_{i}(k+N \mid k+2)\right)\right) \\
& -h_{i}\left(\bar{x}_{i}(k+N \mid k+1), \kappa_{i}\left(\bar{x}_{i}(k+N \mid k+1)\right)\right) \mid \\
& \leq L_{H \kappa_{i}}|| \bar{x}_{i}(k+N \mid k+2)-\bar{x}_{i}(k+N \mid k+1)|| \\
& \leq L_{H \kappa_{i}} L_{f_{i}}^{N-2} \bar{w}
\end{aligned}
$$

Therefore, the bound for $\Delta J_{i 2}^{H}$ is obtained by

$$
\Delta J_{i 2}^{H} \leq-h_{i}\left(x_{i}(k+1), u_{i}^{*}(k+1 \mid k)\right)+L_{S i}(2) \cdot \bar{w}
$$

and so the convergence property is guaranteed when the upper bound is negative. However, we still need to measure $x_{i}(k+1)$ to calculate $h_{i}\left(x_{i}(k+1), u_{i}^{*}(k+1 \mid k)\right)$ and so this is not yet a self-triggered condition. Thus by making use of the bound $\left\|\hat{x}_{i}^{*}(k+1 \mid k)-x_{i}(k+1)\right\| \leq \delta_{i}(k+1)$ in Lemma 2 , and the triangular inequality, we obtain

$$
\left\|x_{i}(k+1)\right\| \geq\left\|\hat{x}_{i}^{*}(k+1 \mid k)\right\|-\delta_{i}(k+1)
$$

Thus we get

$$
\left\|x_{i}(k+1)\right\|^{2} \geq g_{i}^{2}\left(\hat{x}_{i}^{*}(k+1 \mid k), \delta_{i}(k+1)\right)
$$

where $g_{i}\left(x_{i}, \delta_{i}\right)$ is given by $g_{i}\left(x_{i}, \delta_{i}\right)=\max \left\{\left\|x_{i}\right\|-\delta_{i}, 0\right\}$. The bound for the transition cost $h_{i}$ is then given by

$$
\begin{aligned}
& h_{i}\left(x_{i}(k+1), u_{i}^{*}(k+1 \mid k)\right) \\
& \geq \lambda_{\min }(F)\left\|x_{i}(k+1)\right\|^{2}+\left\|u_{i}^{*}(k+1 \mid k)\right\|_{R}^{2} \\
& \geq \lambda_{\min }(F) g_{i}^{2}\left(\hat{x}_{i}^{*}(k+1 \mid k), \delta_{i}(k+1)\right)+\left\|u_{i}^{*}(k+1 \mid k)\right\|_{R}^{2}
\end{aligned}
$$

where $\lambda_{\min }(F)$ is the minimum eigenvalue of the matrix $F$. Thus we obtain

$$
\begin{gathered}
\Delta J_{i 2}^{H} \leq-\lambda_{\min }(F) g_{i}^{2}\left(\hat{x}_{i}^{*}(k+1 \mid k), \delta_{i}(k+1)\right) \\
-\left\|u_{i}^{*}(k+1 \mid k)\right\|_{R}^{2}+L_{S i}(2) \cdot \bar{w}
\end{gathered}
$$

By letting

$$
\begin{gathered}
L_{S i}(2) \cdot \bar{w} \leq \\
\leq \sigma \cdot \lambda_{\min }(F) g_{i}^{2}\left(\hat{x}_{i}^{*}(k+1 \mid k), \delta_{i}(k+1)\right) \\
+\sigma \cdot\left\|u_{i}^{*}(k+1 \mid k)\right\|_{R}^{2}
\end{gathered}
$$


where $0<\sigma<1$, we get $\Delta J_{i 2}^{H}<0$ and then the convergence property is guaranteed without measuring $x_{i}(k+1)$. For guaranteeing the feasibility, we must impose

$$
L_{S i}(3) \leq L_{p i} .
$$

Therefore, the FHOCP is going to be triggered at $k+2$ when either (28) or (29) is violated. Similarly above, we get the following bound of $\Delta J_{i m}^{H}$ for $1<m<N$ :

$$
\begin{aligned}
\Delta J_{i m}^{H} \leq & -h_{i}\left(x_{i}(k+m-1), u_{i}^{*}(k+m-1 \mid k)\right) \\
& +L_{S i}(m) \cdot \bar{w} .
\end{aligned}
$$

From Lemma 2, we obtain

$$
\begin{gathered}
\Delta J_{i m}^{H} \leq-\lambda_{\min }(F) g_{i}^{2}\left(x_{i}^{*}(k+m-1 \mid k), \delta_{i}(k+m-1)\right) \\
-\left\|u_{i}^{*}(k+m-1 \mid k)\right\|_{R}^{2}+L_{S}(m) \cdot \bar{w}
\end{gathered}
$$

Thus the self-triggered condition is given by

$$
\begin{aligned}
& L_{S i}(m) \cdot \bar{w}-\sigma \cdot\left\|u_{i}^{*}(k+m-1 \mid k)\right\|_{R}^{2} \\
& \leq \sigma \cdot \lambda_{\min }(F) g_{i}^{2}\left(x_{i}^{*}(k+m-1 \mid k), \delta_{i}(k+m-1)\right)
\end{aligned}
$$

The additional condition for guaranteeing the feasibility is

$$
L_{S i}(m+1) \leq L_{p i}
$$

Therefore, the following self-triggered condition is formulated;

(Self-triggered MPC): Assume that the FHOCP is solved at $k$. Then, the FHOCP is triggered at $k+1$ when either (25) or (26) is violated. For $k+m$ where $1<m<N$, the FHOCP is triggered at $k+m$, when either (31) or (32) is violated. When it is satisfied for all $1 \leq m<N$, the FHOCP is solved at $k+N$.

Remark 3. Clearly, our proposed self-triggered conditions are more restrictive than the event-based case, since we need to take into account the maximum difference or triangular relation between predictive states and the actual states (27), as well as (32) for guaranteeing the feasibility. This conservativeness can also be seen in our simulation example given in section 6.

Definition 2 (Information vector for self-triggered case). When the FHOCP is solved at $k$, the information vector transmitted from $i$ is the same as in the event-based case;

$$
\boldsymbol{z}_{i}(k)=\left\{\hat{x}_{i}^{*}(k+1 \mid k), \cdots, \hat{x}_{i}^{*}(k+N \mid k), f_{i}\left(\hat{x}_{i}^{*}(k+N \mid k), \kappa_{i}(\cdot)\right)\right\} .
$$

When it is not solved, the information is different from one in event-based case, as it does not measure the actual states. Let $\tilde{\boldsymbol{x}}_{i}(k)=\left\{\tilde{x}_{i}(k+l \mid k-n)\right\}_{l=0}^{N}$ be the predictive states based on $\tilde{x}_{i}(k \mid k-n)=\hat{x}_{i}^{*}(k \mid k-n)$ given that the FHOCP is solved at $k-n$, and $\overline{\boldsymbol{u}}_{i}(k)$. Then, the information vector for self-triggered case is given by

$$
\begin{aligned}
\boldsymbol{z}_{i}(k+1)= & \left\{\tilde{x}_{i}(k+1 \mid k-n), \cdots,\right. \\
& \left.\tilde{x}_{i}(k+N \mid k-n), \tilde{x}_{i}(k+N+1 \mid k-n)\right\}
\end{aligned}
$$

where $\tilde{x}_{i}(k+N+1 \mid k-n)=f_{i}\left(\tilde{x}_{i}(k+N \mid k-n), \kappa_{i}(\cdot)\right)$. Clearly, this information vector is less accurate than the event-based case where the information is calculated based on the current state measurement, thus we may need to restrict the triggering rule by tuning $\sigma$ in (31) to transmit more reliable information. 


\subsection{Feasibility analysis}

In this section the feasibility analysis for self-triggered NMPC for each agent is going to be given. By imposing more restricted bounds for $\bar{w}$ than in the event-based case, we are able to show the feasibility.

Theorem 2. Let the system be described by (2), and assume that Assumptions 1-7 are satisfied. Then, the FHOCP under the self-triggered control is feasible if the uncertainties are bounded by

$$
\bar{w} \leq \frac{\left(L_{f_{i}}-1\right)\left(\alpha_{i}-\alpha_{v_{i}}\right)}{L_{V_{i}}\left(L_{f_{i}}^{N}-1\right)}
$$

Proof. The feasibility is going to be shown in the same way as in the event-based case.

1. $\overline{\boldsymbol{u}}_{i}(k+m) \in U_{i}$

This is clearly admissible from the expression of $\bar{u}_{i}(k+l \mid k+m)$ given by (23).

2. $\bar{x}_{i}(k+l \mid k+m) \in \mathcal{X}_{i}^{l-m}$ for $l=m+1, \cdots, N$.

Since $\bar{u}_{i}(k+l \mid k+m)=u_{i}^{*}(k+l \mid k)$ for $l=m, \cdots, N-1$, from Lemma 2 , we get $\bar{x}_{i}(k+l \mid k+m) \in \mathcal{X}_{i}^{l-m}$.

3. $\bar{x}_{i}(k+m+N \mid k+m) \in \mathcal{X}_{f_{i}}$.

First we show $\bar{x}_{i}(k+N \mid k+m) \in \Phi_{i}$. By using

$$
\left\|\bar{x}_{i}(k+N \mid k+m)-\hat{x}_{i}^{*}(k+N \mid k)\right\| \leq L_{f_{i}}^{N-m} \frac{L_{f_{i}}^{m}-1}{L_{f_{i}}-1} \bar{w}
$$

and $\hat{x}_{i}^{*}(k+N \mid k) \in \mathcal{X}_{f_{i}}$, we get

$$
\begin{aligned}
V_{i}\left(\bar{x}_{i}(k+N \mid k+m)\right) & \leq \alpha_{v_{i}}+L_{V_{i}} L_{f_{i}}^{N-m} \frac{L_{f_{i}}^{m}-1}{L_{f_{i}}-1} \bar{w} \\
& \leq \alpha_{v_{i}}+L_{V_{i}} \frac{L_{f_{i}}^{N}-1}{L_{f_{i}}-1} \bar{w} \leq \alpha_{i}
\end{aligned}
$$

where the last inequality is obtained from (35). Hence, $\bar{x}_{i}(k+N \mid k+m) \in \Phi_{i}$. Furthermore, by Assumption 3,

$$
\begin{aligned}
V_{i}\left(\bar{x}_{i}(k+N+1 \mid k+m)\right) & \leq V_{i}\left(\bar{x}_{i}(k+N \mid k+m)\right) \\
& \leq \alpha_{i} .
\end{aligned}
$$

Thus $\bar{x}_{i}(k+N+1 \mid k+m) \in \Phi_{i}$. By recursion, we get $\bar{x}_{i}(k+N+2 \mid k+m) \in \Phi_{i}$, $\cdots, \bar{x}_{i}(k+N+m-1 \mid k+m) \in \Phi_{i}$, and thus $\bar{x}_{i}(k+N+m \mid k+m) \in \mathcal{X}_{f_{i}}$.

4. $\bar{x}_{i}(k+l \mid k+m) \in \mathcal{X}_{i}^{l-m}$ for $l=N+1, \cdots, N+m-1$.

By using Assumption $3, \bar{x}_{i}(k+N \mid k+m) \in \Phi_{i} \subseteq \mathcal{X}_{i}^{N-m}$, and

$$
\begin{array}{lll}
\bar{x}_{i}(k+N+1 \mid k+m) & \in \Phi_{i} \subseteq \mathcal{X}_{i}^{N-m+1} \\
\bar{x}_{i}(k+N+2 \mid k+m) & \in \Phi_{i} \subseteq \mathcal{X}_{i}^{N-m+2} \\
& \vdots & \\
\bar{x}_{i}(k+m+N-1 \mid k+m) & \in \Phi_{i} \subseteq \mathcal{X}_{i}^{N-1}
\end{array}
$$

Therefore, $\bar{x}_{i}(k+l \mid k+m) \in \mathcal{X}_{i}^{l-m}$ for $l=N+1, \cdots, N+m-1$. 
5. $\bar{J}_{i}^{H}(k+m) \leq \gamma_{i}(k+m)$

For $m=1$, by using (24), we get

$$
\begin{aligned}
\bar{J}_{i}^{H}(k+1) & \leq J_{i}^{H *}(k)-h_{i}\left(x_{i}(k), u_{i}^{*}(k \mid k)\right)+L_{p i} \cdot \bar{w} \\
& =\gamma_{i}(k+1)
\end{aligned}
$$

where $L_{S i}(1)=L_{p i}$ is used. Hence, the cost follows $\bar{J}_{i}^{H}(k+1) \leq \gamma_{i}(k+1)$ at $m=1$. For $1<m<N$, when the FHOCP is triggered at $k+m, L_{S i}(m) \leq L_{p i}$ has been satisfied from the self-triggering rule (32) previously at $k+m-1$. Thus we get

$$
\begin{aligned}
\bar{J}_{i}^{H}(k+m) \leq & \bar{J}_{i}^{H}(k+m-1)+L_{p i} \cdot \bar{w} \\
& -h_{i}\left(x_{i}(k+m-1), u_{i}^{*}(k+m-1 \mid k)\right) \\
= & \gamma_{i}(k+m)
\end{aligned}
$$

Hence the cost satisfies $\bar{J}_{i}^{H}(k+m) \leq \gamma_{i}(k+m)$, so the feasibility for the last constraint is guaranteed for $m=1, \cdots, N-1$. This finally completes the proof for guaranteeing the feasibility.

Remark 4. In order to calculate $\gamma_{i}(k+m)$ when the FHOCP is solved at $k+m$, the previous actual state $x_{i}(k+m-1)$ needs to be measured in order to calculate $\bar{J}_{i}^{H}(k+m-1)$ in $\gamma_{i}(k+m)$ even in the case of self-triggered control.

Remark 5. One can see that the bound for the disturbance can be very restrictive due to the term $\left(L_{f_{i}}^{N}-1\right) /\left(L_{f_{i}}-1\right)=1+L_{f_{i}}^{1}+L_{f_{i}}^{2}+\cdots+L_{f_{i}}^{N-1}$ in the denominator, compared with $L_{f_{i}}^{N-1}$ for the event-based case. This can be made less restrictive by setting the maximum next triggering step $m_{\max }$. We can then get the new bound for the disturbance as

$$
\bar{w} \leq \frac{\left(L_{f_{i}}-1\right)\left(\alpha_{i}-\alpha_{v_{i}}\right)}{L_{V_{i}}\left(L_{f_{i}}^{N}-L_{f_{i}}^{N-m_{\max }}\right)}
$$

This makes the self-triggering condition more conservative, though we allow larger disturbance.

\section{Stability Analysis}

In this section the stability analysis for MPC under the event-based and self-triggered scheme is going to be given. According to [23], it is proven for a single agent that the closed loop system under the standard MPC scheme is Input-to-State Stable (ISS) with respect to bounded disturbances, and that the trajectory of the state evolves towards inside $\Phi_{i}$.

In this paper, we will show that $J_{i}^{H}(k)$ is an ISS Lyapunov function, and the same stability property is guaranteed as in [23] for both event-based and self-triggered case by making use of the additional constraint imposed on the FHOCP. Referring to [23], $J_{i}^{H}(k)$ is said to be an ISS Lyapunov function for agent $i$, if the following is satisfied; 
1. $J_{i}^{H}(k) \geq \alpha_{1}\left(x_{i}(k)\right), \forall x_{i}(k) \in \mathcal{X}_{i}$, and $J_{i}^{H}(k) \leq \alpha_{2}\left(x_{i}(k)\right) \forall x_{i}(k) \in \Phi_{i}$, where $\alpha_{1}$ and $\alpha_{2}$ are $\mathcal{K}_{\infty}$ functions.

2. $J_{i}^{H}(k+1)-J_{i}^{H}(k) \leq-\alpha_{3}\left(x_{i}(k)\right)+\sigma(\|w\|), \forall x_{i}(k) \in \mathcal{X}_{i}$, where $\alpha_{3}$ is a $\mathcal{K}_{\infty}$ function, and $\sigma$ is a $\mathcal{K}$-function.

where 1) is obtained in the same way as in [23]. Before showing 2), we must note that $J_{i}^{H}(k)$ is not always the cost from the optimal control inputs, and thus this takes values in two different ways : when the FHOCP is solved, $J_{i}^{H *}(k)=J_{i}^{H}\left(\boldsymbol{x}_{i}^{*}(k), \boldsymbol{u}_{i}^{*}(k)\right)$ and when not solved, $\bar{J}_{i}^{H}(k)=J_{i}^{H}\left(\overline{\boldsymbol{x}}_{i}(k), \overline{\boldsymbol{u}}_{i}(k)\right)$. According to the triggering rules, we have already shown 2) for the differences $\bar{J}_{i}^{H}(k+1)-J_{i}^{H *}(k)$ and $\bar{J}_{i}^{H}(k+1)-\bar{J}_{i}^{H}(k)$, i.e., when the triggering conditions are satisfied at $k+1$. Therefore, we need to check the difference when the FHOCP is solved at $k+1$, i.e., $J_{i}^{H *}(k+1)-J_{i}^{H *}(k)$ and $J_{i}^{H *}(k+1)-\bar{J}_{i}^{H}(k)$ for guaranteeing the ISS property. This is easily shown, since by using the last constraint $J_{i}^{H}(k+1) \leq \gamma_{i}(k+1)$ when the FHOCP is solved at $k+1$, we get from (8) that

$$
\begin{aligned}
J_{i}^{H *}(k+1)-J_{i}^{H *}(k) & \leq-h_{i}\left(x_{i}(k), u_{i}^{*}(k \mid k)\right)+L_{p i} \cdot \bar{w} \\
& \leq-\lambda_{\min }(F)\left\|x_{i}(k)\right\|^{2}+\sigma(\|w\|)
\end{aligned}
$$

where $J_{i}^{H *}(k)$ can be replaced with $\bar{J}_{i}^{H}(k)$. Therefore, from [23], it is proved that $J_{i}^{H}(k)$ is an ISS Lyapunov function, and the following stability result is concluded.

Theorem 3. Let the system be given by (2), and assume that all the Assumptions 1 to 5 , and in addition 6 and 7 for self-triggered case, are satisfied. Furthermore, assume that the additive disturbance is bounded by (21) for the event-based case and (35) for the self-triggered case. The control update is given under either event-based or selftriggered condition, which are described above. Then, the system for agent $i$ is ISS and thus the trajectory of the state reaches toward inside $\Phi_{i}$ where it is ultimately bounded.

\section{Simulation Results}

The simulation results of the proposed methods are given in this section. Consider the position control of two non-holonomic agents having the same dynamics in two dimensions, where the nominal model of each agent is given by

$$
\left\{\begin{array}{ccc}
x_{i}(k+1) & = & x_{i}(k)-v_{i}(k) T \cos \theta_{i}(k) \\
y_{i}(k+1) & = & y_{i}(k)-v_{i}(k) T \sin \theta_{i}(k) \\
\theta_{i}(k+1) & = & \theta_{i}(k)-\omega_{i}(k) T
\end{array}\right.
$$

which is simply denoted as $\chi_{i}(k+1)=f_{i}\left(\chi_{i}(k), u_{i}(k)\right)$, where $\chi_{i}=\left[x_{i}, y_{i}, \theta_{i}\right]^{\top}$ for $i=1,2$ denotes the state vector consisting of the position of $i$-th robot and its direction $\theta_{i} . u=\left[v_{i}, \omega_{i}\right]^{\top}$ is the control input and the constraints are given by $\left|v_{i}\right| \leq \bar{v}=2.5$ and $\left|\omega_{i}\right| \leq \bar{\omega}=0.5 . T=0.2$ is the sampling time. The matrices for the transition cost are given by $F=0.5 I_{3}, R=0.1 I_{2}$, and the coupling costs are $Q_{12}=0.5 I_{2}, Q_{21}=$ $1.0 I_{2}$. The prediction horizon is set to $N=35$ steps. The terminal cost is given by $V_{i}=\chi_{i}^{\top} \chi_{i}$, and the parameters $\alpha_{i}$ and $\alpha_{v_{i}}$ for defining this terminal region are given by 


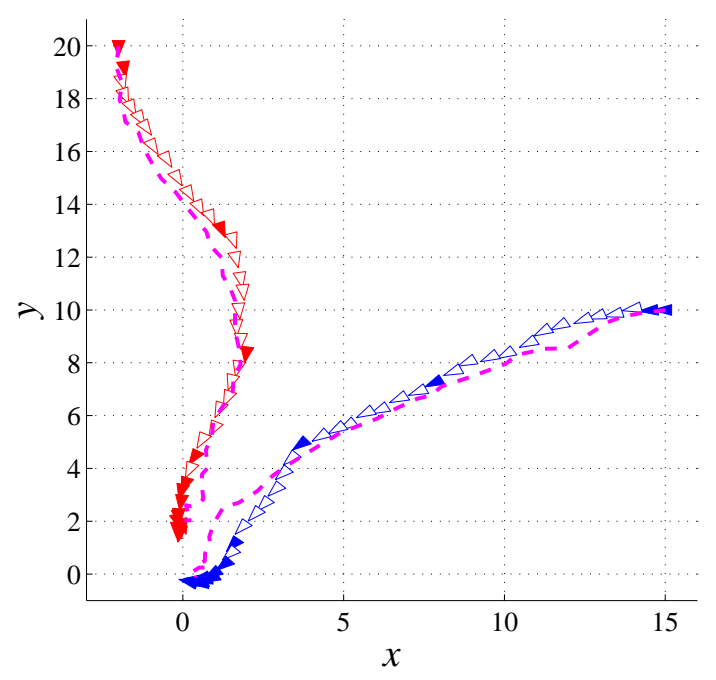

Figure 1: Trajectory of two agents under the several schemes with additive disturbances $\bar{w}=0.25$ : the solid line represents the trajectory of the agents under event-based MPC, and the dashed line represents the trajectory under Standard MPC.

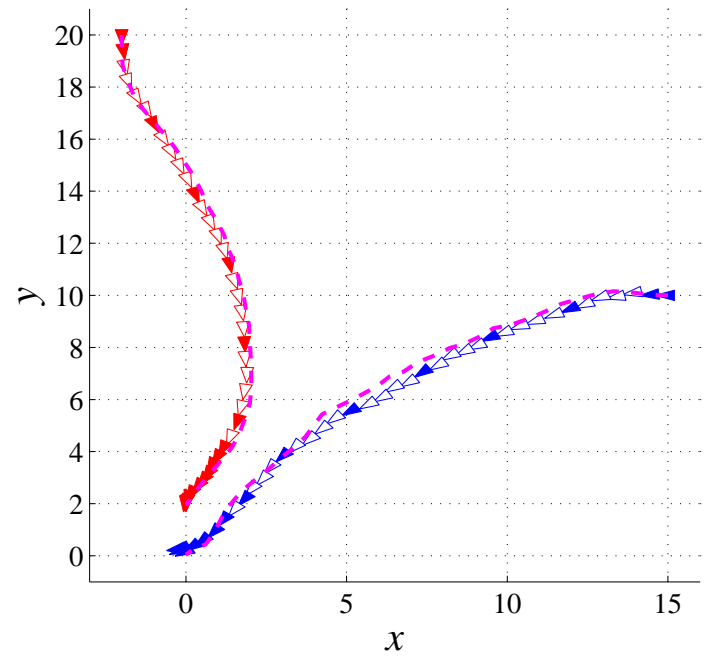

Figure 2: Trajectory of two agents under the several schemes with additive disturbances $\bar{w}=0.05$ : the triangles represent the trajectory of the agents under self-triggered MPC, and the dashed line represents the trajectory under Standard MPC. The filled triangles show the points when the FHOCP is triggered. 
$\alpha_{i}=2.25$ and $\alpha_{v_{i}}=0.68$ according to the procedure in [22]. With computed Lipschitz constants $L_{f_{i}}=1.01, L_{V_{i}}=4.50$, the allowable disturbance for guaranteeing the feasibility is $\bar{w}=0.25$ for event-based case and $\bar{w}=0.052$ for self-triggered case with $m_{\max }=5$. Note that the size of disturbances for the self-triggered case becomes much smaller than the event-based case as pointed out in Remark 5, although this could be less conservative by changing the terminal region (or $L_{V_{i}}$ ), or the prediction horizon. The initial points of the agents are $x_{1}(0)=[-220-\pi / 2]^{\top}, x_{2}(0)=[10,15, \pi]^{\top}$, and their goals are $x_{\text {goal1 }}=[0,2,-\pi / 2]^{\top}, x_{\text {goal2 }}=[0,0, \pi]^{\top}$. Thus the desired distance vector is $d_{21}=[0,2]^{\top}$ and $d_{12}=[0,-2]^{\top}$. Fig. 1 shows the trajectory of two agents
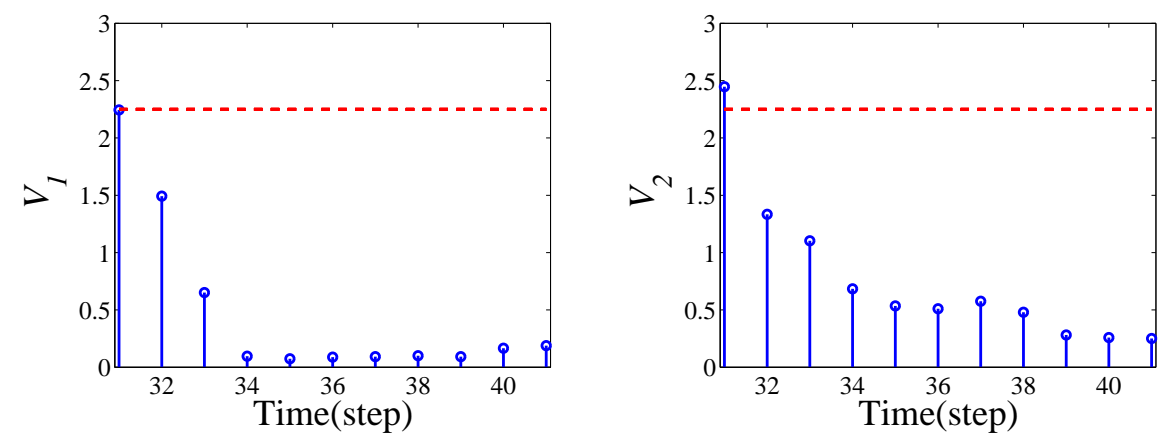

Figure 3: The sequence of $V_{1}$ under event- Figure 4: The sequence of $V_{2}$ under eventbased case. Red dot-line is the boundary of based case. Red dot-line is the boundary of the terminal region $V_{1}=\alpha_{1}$. the terminal region $V_{2}=\alpha_{2}$.
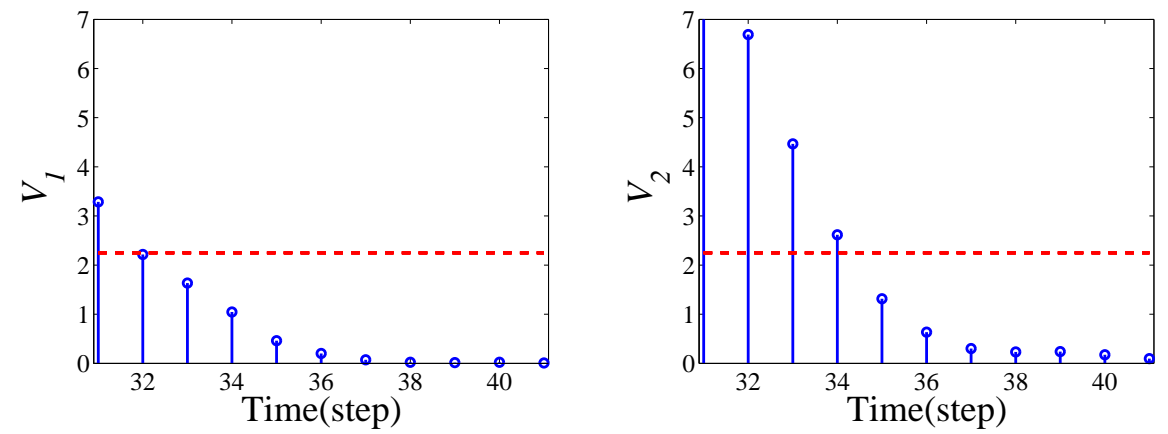

Figure 5: The sequence of $V_{1}$ under self- Figure 6: The sequence of $V_{2}$ under selftriggered case. Red dot-line is the boundary triggered case. Red dot-line is the boundary of the terminal region $V_{1}=\alpha_{1}$. of the terminal region $V_{2}=\alpha_{2}$.

under event-based and standard MPC schemes with allowable size of disturbances $\bar{w}=$ 0.25, and Fig. 2 shows the trajectory under self-triggered and standard MPC schemes with $\bar{w}=0.05$. As shown in Fig. 1 and Fig. 2, both agents can reach to the desired goals under all of these control methods. 
To verify the stability property, we plot the sequence of $V_{1}\left(\chi_{1}\right)$ and $V_{2}\left(\chi_{2}\right)$ for the last 10 steps in Fig. 3, Fig. 4 for event-based case and Fig. 5, Fig. 6 for self-triggered case. For both cases, $V_{1}$ and $V_{2}$ converge to the terminal region $V_{i} \leq \alpha_{i}$, which verifies the convergence property analyzed in section 5 .

Fig. 7 shows the triggering instants of the one agent under event-based and selftriggered control, where if the value is 1 the FHOCP is triggered and if 0 the FHOCP is not triggered. We can see from Fig. 7 that the FHOCP is aperiodically solved, and therefore we can conclude that the reduction of over-all energy consumption and the usage of communication resources can be achieved. Note that the triggering instants become more frequent as each agent approaches its goal and completely periodic for the last 10 time steps. This is because the right hand side of (18) (or (31)) becomes smaller as the state $x_{i}(k)$ approaches the origin, and the effect of the disturbance term in the left hand side becomes relatively large. This problem might be avoided by applying dual-mode MPC, where the local controller is used in the terminal region, see for example, [18].

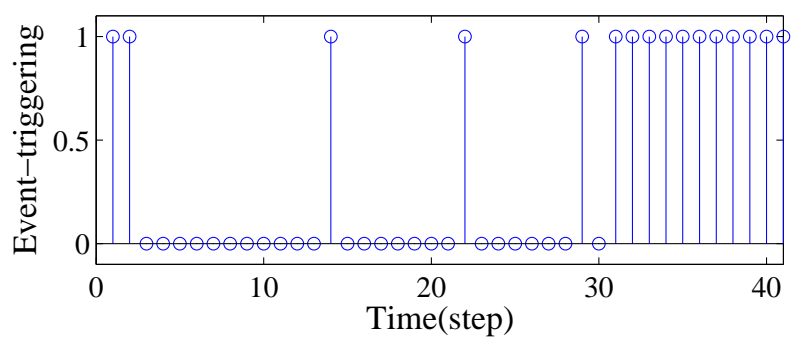

(a) Event-based case

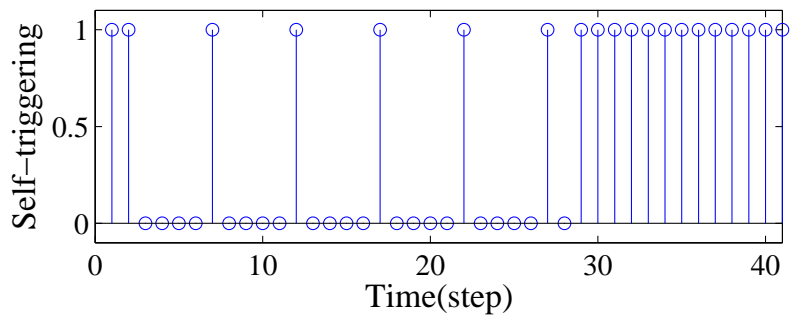

(b) Self-triggered case

Figure 7: Triggering instants for one agent under event-based (upper) and self-triggered (below). The MPC is triggered when the values are 1 


\section{Conclusions}

We proposed event-based and self-triggered MPC for distributed agents having nonlinear dynamics with additive bounded disturbances. In these control methods, each agent aperiodically solves FHOCP in order not only to reduce the energy consumption but also to reduce the communication loads, while we can still guarantee the stability and feasibility. Finally our proposal was verified by a simple simulation result by showing that all agents cold reach to the terminal region.

\section{References}

[1] Eqtami, A. , Dimarogonas, D. V., and Kyriakopoulos, K. J.: 'Event-Based Model Predictive Control for the Cooperation of Distributed Agents', American Control Conf., 2012, pp. 6473 - 6478

[2] Eqtami, A., Dimarogonas, D. V., and Kyriakopoulos, K. J.: 'Event-triggered strategies for decentralized model predictive controllers', IFAC World Congress, 2011, pp. $10068-10073$

[3] Eqtami, A., Heshmati-Alamdari, S., Dimarogonas, D. V., and Kyriakopoulos, K. J.: 'Self-triggered Model Predictive Control for nonholonomic systems', European Control Conf., 2013, pp. 638 - 643

[4] Eqtami, A., Heshmati, S., Dimarogonas, D. V. and Kyriakopoulos, K. J.: 'SelfTriggered Model Predictive Control Framework for the Cooperation of Distributed Nonholonomic Agents', The 52nd IEEE Conf. on Decision and Control, 2013, pp. $7384-7389$

[5] Seyboth, G. S., Dimarogonas, D. V. and Johansson K. H.: 'Event-based broadcasting for multi-agent average consensus', Automatica, 2013, 49, (1), pp. 245 252

[6] Heemels, W. P. M. H., Johansson, K. H., and Tabuada, P.: 'An Introduction to Event-triggered and Self-triggered Control', The 51st IEEE Conf. on Decision and Control, 2012, pp. 3270 - 3285

[7] Heemels, W. P. M. H. and Donkers, M. C. F.: 'Model-based periodic eventtriggered control for linear systems', Automatica, 2013, 49, (3), pp. 698 - 711

[8] Gommans, T., Antunes, D., Donkers, T., et al.: 'Self-triggered linear quadratic control', Automatica, 2014, 50, (4), pp. 1279 - 1287

[9] Lehmann, D., Henriksson, E., and Johansson, K. H.: 'Event-Triggered Model Predictive Control of Discrete-Time Linear Systems subject to Disturbances', European Control Conf., 2013, pp. 1156 - 1161

[10] Li, H. and Shi, Y.: 'Event-triggered robust model predictive control of continuous-time nonlinear systems', Automatica, 2014, 50, (5), pp. 1507 - 1513 
[11] Li, H. and Shi, Y.: 'Distributed model predictive control of constrained nonlinear systems with communication delays', Systems and Control Letters, 2013, 62, (10), pp. $819-826$

[12] Chen, W. and Chong, J.: 'Distributed Model Predictive Control of Dynamically Decoupled Linear Systems with Coupled Cost', IEEE Conf. Decision and Control and 28th Chinese Control Conf., 2009, pp. 5420 - 5425

[13] Chen W. and Chong, J. : 'Dynamically Decoupled Non-Linear Systems with Coupled Cost: a Distributed Model Predictive Control Approach', The 8th Asian Control Conference, 2011, pp. 1376 - 1380

[14] Marruedo, D.L., Alamo, T. and Camacho, E. F.: 'Input-to-state stable MPC for constrained discrete-time nonlinear systems with bounded additive uncertainties', The 41st IEEE Conf. Decision and Control, 2002, pp. 4619 - 4624

[15] Varutti, P., Kern, B., Faulwasser, T., and Findeisen, R.: 'Event-based Model Predictive Control for Networked Control Systems', The 48th IEEE Conf. Decision and Control and 28th Chinese Control Conf., 2009, pp. 567 - 572

[16] Dunbar, W. B. and Murray, R. M.: 'Distributed Receding Horizon Control for Multi- Vehicle Formation Stabilization', Automatica, 2006, 42, (4), pp. 549 - 558

[17] Berglind, J. D. J. B., Gommans, T. M. P and Heemels, W. P. M. H.: 'Self-triggered MPC for constrained linear systems and quadratic costs', The 4th IFAC Nonlinear Model Predictive Control Conf., 2012, pp. 342 - 348

[18] Michalska, H. and Mayne D. Q. Mayne: 'Robust receding horizon control of constrained nonlinear systems', IEEE Trans. Autom. Control, 1993, 38, (11), pp. $1623-1633$

[19] Richards A. and How, J.: 'Decentralized Model Predictive Control of Cooperating UAVs', The 43rd IEEE Conf. on Decision and Control, 2004, pp. 4286 - 4291

[20] Keviczky, T., Borrelli, F., Fregene, K., Godbole, D., and Balas, G. J.: 'Decentralized Receding Horizon Control and Coordination of Autonomous Vehicle Formations', IEEE Trans. Control Systems Technology, 2008, 16, (1), pp. 19 - 33

[21] Franco, E., Parisini, T., and Polycarpou, M. M.: 'Cooperative Control of Distributed Agents with Nonlinear Dynamics and Delayed Information Exchange: a Stabilizing Receding-Horizon Approach', The 44th IEEE Conf. on Decision and Control and the European Control Conf., 2005, pp. 2206 - 2211

[22] Zhu, Y. and Ozuner, U.: 'Robustness Analysis on Constrained Model Predictive Control for Nonholonomic Vehicle Regulation', American Control Conf., 2009, pp. $3896-3901$

[23] Magni, L., Raimondo, D.M., and Scattolini, R.: 'Input-to-State Stability for Nonlinear Model Predictive Control', The 45th IEEE Conf. on Decision and Control, 2006, pp. 4836 - 4841 
[24] Åström, K. J. and Bernhardsson, B. M.: 'Comparison of periodic and event based sampling for first order stochastic systems', IFAC World Conf., 1999, pp. 301 306

[25] Tabuada, P.: 'Event-triggered real-time scheduling of stabilizing control tasks', IEEE Trans. on Autom. Control, 2007, 52, (9), pp. 1680 - 1685

[26] Mazo, M. Jr., Anta, A., and Tabuada, P.: 'An ISS self-triggered implementation of linear controllers', Automatica, 2010, 46, (8), pp. 1310 - 1314

[27] Stewart, B., Wright, S., and Rawlings, J. B.: 'Cooperative distributed model predictive control for nonlinear systems', Journal of Process Control, 2011, 21, (5), pp. $698-704$

[28] Liu, S., Zhang, J., Feng, Y., et al.: 'Distributed Model Predictive Control with Asynchronous Controller Evaluations', The Canadian Journal of Chemical Engineering, 2013, 91, pp. 1609 - 1620 